\title{
Experiencias de los estudiantes exitosos de primera generación en la educación superior mexicana: un estudio narrativo
}

\author{
Experiences of Successful First-Generation Students in Mexican Higher \\ Education: A narrative study
}

\section{Experiências de estudantes de primeira geração bem-sucedidos no ensino superior mexicano: um estudo narrativo}

\author{
Enrique Bonilla Murillo \\ Universidad Autónoma de Tamaulipas, México \\ ebonilla@docentes.uat.edu.mx \\ https://orcid.org/0000-0002-3623-0989 \\ Verónica Sanité Solís Herebia \\ Universidad Autónoma de Tamaulipas, México \\ vsolis@docentes.uat.edu.mx \\ http://orcid.org/0000-0001-9070-085X
}

\section{Resumen}

La diversidad de la población estudiantil dificulta a la universidad poder satisfacer la demanda educativa. Los estudiantes de primera generación (EPG) pertenecen a una población vulnerable por sus características y retos de mantenerse en su trayectoria escolar. Los EPG son los primeros en sus familias en asistir a una universidad; por lo tanto, enfrentan retos complejos como no conocer la cultura escolar, tener bajo rendimiento académico, dificultades económicas y típicamente no tienen un proyecto académico. El propósito de este estudio, por tanto, fue conocer a través de sus historias las experiencias de EPG exitosos en la educación mexicana. Se utilizó un método cualitativo con enfoque narrativo. Se colectaron datos de cuatro participantes a través de entrevistas narrativas de profundidad para encontrar cuáles fueron sus retos, sus sistemas de apoyo 


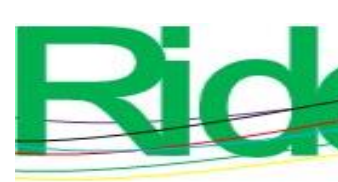

Revista Iberoamericana para la Investigación y el Desarrollo Educativo ISSN $2007-7467$

y sobre todo su motivación hasta alcanzar el éxito. Las historias narradas demuestran que la visión clara sobre lo que quieren profesionalmente y habilidades emocionales como la motivación y la resiliencia les permitieron conseguir sus metas académicas. Sin embargo, cabe resaltar que no todo debe depender de los EPG, pues la universidad tiene la obligación de proveer el apoyo que los estudiantes necesitan de acuerdo a sus necesidades.

Palabras clave: enseñanza superior, estudiante universitario, sociología del estudiante.

\section{Abstract}

The diversity of the student population makes it difficult for the university to meet the educational demand. First-generation students (EPG) belong to a vulnerable population due to their characteristics and challenges of staying in their school careers. EPGs are the first in their families to attend college; therefore, they face complex challenges such as not knowing the school culture, having poor academic performance, financial difficulties, and typically do not have an educational project. This study aimed to understand the experiences of successful EPGs in Mexican education through their stories. A qualitative method with a narrative approach was used. Data were collected from four participants through in-depth narrative interviews to find out their challenges, support systems, and motivation that allowed them to continue until they reach success. The narrated stories demonstrate that a clear vision of what they want professionally and emotional skills such as motivation and resilience enable them to achieve their academic goals. However, it depends not only on the EPGs; the university must provide the students' support according to their needs.

Keywords: higher education, student sociology, university student.

\section{Resumo}

A diversidade da população estudantil dificulta o atendimento da demanda educacional da universidade. Os alunos da primeira geração (EPG) pertencem a uma população vulnerável devido às suas características e desafios de permanência na carreira escolar. EPGs são os primeiros em suas famílias a frequentar a faculdade; portanto, enfrentam desafios complexos como não conhecer a cultura escolar, ter baixo desempenho acadêmico, dificuldades financeiras e, normalmente, não ter um projeto acadêmico. O objetivo deste estudo, portanto, foi conhecer por meio de suas histórias as experiências de EPG de sucesso na educação mexicana. Foi utilizado um método qualitativo com abordagem narrativa. Os dados foram coletados de quatro participantes por meio de entrevistas narrativas em profundidade para descobrir quais eram seus desafios, seus sistemas 


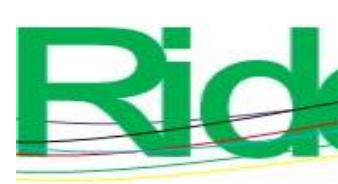

Revista Iberoamericana para la Investigación y el Desarrollo Educativo ISSN $2007-7467$

de apoio e, acima de tudo, sua motivação para alcançar o sucesso. As histórias contadas mostram que a visão clara do que desejam profissionalmente e as competências emocionais, como motivação e resiliência, permitiram que atingissem seus objetivos acadêmicos. No entanto, devese destacar que nem tudo deve depender dos EPGs, pois a universidade tem a obrigação de fornecer o suporte de que os alunos precisam de acordo com suas necessidades.

Palavras-chave: ensino superior, estudante universitário, sociologia estudantil. Fecha Recepción: Abril 2021 Fecha Aceptación: Octubre 2021

\section{Introducción}

Los retos de la educación superior tanto pública como privada a nivel mundial son diversos, aunque algunos se relacionan con el financiamiento, la gobernabilidad, la rendición de cuentas, la transparencia, los costos elevados de las colegiaturas y la retención de los estudiantes. La tarea pendiente de la universidad, por ende, es ofertar programas de educación superior flexibles y adecuados a las necesidades y características de los estudiantes del siglo XXI, quienes demandan una formación creativa e innovadora para acceder a mejores puestos de trabajos (Dennis, 2012).

En este contexto, la tecnología juega un papel preponderante porque permite proveer una educación más flexible. Sin embargo, se debe tomar en cuenta que el uso de esas herramientas por sí mismas resultaría insuficiente si no se cuenta también con sistemas de apoyo que orienten y motiven el ingreso y permanencia de los estudiantes. Por ello, surgen preguntas como las siguientes: ¿qué estrategias emplea la educación superior para garantizar el éxito de los estudiantes? y ¿qué programas de apoyo benefician la permanencia de los estudiantes?

El éxito de los alumnos depende de múltiples factores, entre ellos la creación de ambientes educativos que beneficien sus experiencias académicas, lo cual puede impactar en sus percepciones y, en consecuencia, en su éxito o fracaso escolar.

Por ese motivo, el propósito del presente estudio cualitativo fue comprender las experiencias de estudiantes exitosos de primera generación en educación superior a través de la narración de sus historias de vida. De esta manera, se comprenderá cómo impactan los sistemas de apoyo universitario y cómo contribuyen sus historias de vida en el éxito académico en la educación superior. 


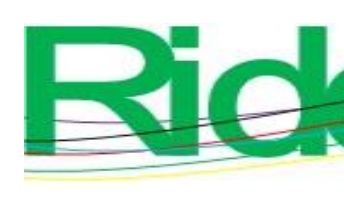

Revista Iberoamericana para la
Investigación y el Desarrollo Educativo
ISSN $2007-7467$

\section{Revisión de literatura}

Los estudiantes de primera generación (EPG) pertenecen a un grupo de la población estudiantil de la educación superior que tienen características y necesidades únicas. En Estados Unidos, por ejemplo, más de $20 \%$ de la población estudiantil está compuesta por EPG y solo $11 \%$ logra obtener un grado universitario, en comparación con $55 \%$ de estudiantes tradicionales (Katrevich y Aruguete, 2017).

Típicamente, los EPG no conocen la cultura universitaria porque son los primeros miembros de sus familias que se matriculan en una institución de educación superior, es decir, sus padres no cuentan con un título universitario. Los EPG son estudiantes sin proyecto escolar definido (Soto Hernández, 2016). Entre otras características, los EPG son alumnos no tradicionales, pues son de mayor edad en comparación a los tradicionales, con trayectorias escolares no lineales, y tienen hijos y trabajan (Ishitani, 2016; Linne, 2018). De hecho, en muchos casos los EPG son mujeres responsables económicamente de sus familias (Linne, 2018), de ahí que se enfrenten a dificultades muy diversas y particulares.

\section{Carencias emocionales y académicas}

En primer lugar, los EPG son estudiantes con necesidades emocionales que afectan su desempeño y permanencia en la educación superior. En estudios sobre las cargas emocionales de estudiantes latinos en EE. UU., Tello y Lonn (2017) sugirieron que estos alumnos requieren de estrategias para sobrellevar el estrés que genera un ambiente universitario. Por eso, deben desarrollar inteligencia emocional y cultural, resiliencia y motivación intrínseca, así como habilidades de liderazgo que les permitan visualizar y planificar su futuro (Jacobson-Lundeberg, 2016; Shumaker y Wood, 2016; Soria, Roberts y Reinhard, 2015; Tello y Lonn, 2017).

Asimismo, en una investigación sobre el capital social de estudiantes indocumentados en EE. UU., Montiel (2016) explica que los EPG son estudiantes que necesitan habilidades de resiliencia para ingresar y mantenerse matriculados en la universidad. Por otro lado, en estudios en Chile, Soto Hernández (2016) comenta que dichos estudiantes se sienten excluidos por desconocer el ambiente universitario.

Para los EPG, la inteligencia emocional es un factor clave para el desempeño académico y para la integración social en la universidad. Aunado a los factores emocionales, los EPG en muchos casos son estudiantes con limitadas habilidades académicas que ponen en riesgo su permanencia en la educación superior. Las calificaciones bajas y las pocas habilidades en matemáticas y en 


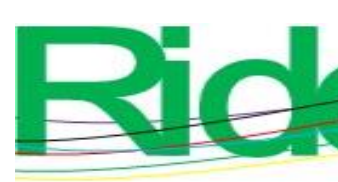

Revista Iberoamericana para la Investigación y el Desarrollo Educativo ISSN $2007-7467$

pensamiento crítico son un reto más para los EPG (Katrevich y Aruguete, 2017). Además, los EPG tienen una autopercepción baja sobre sus habilidades y desempeño escolar (Barraza-López y González, 2016; Flanagan, 2016).

\section{Bajos recursos económicos}

Los EPG comparten otra característica que limita su desempeño académico. Diferentes estudios describen a los EPG como estudiantes de bajos recursos económicos (Hirudayaraj y Maclean, 2018; Ishitani, 2016; Linne, 2018; Montiel, 2017). Carecer de ingresos no solo afecta el estado emocional de los estudiantes, sino que principalmente les deja menos tiempo para estudiar, puesto que tienen que trabajar para satisfacer sus necesidades familiares, personales y académicas.

\section{La familia y la educación universitaria}

Sentirse apoyado por la familia es un factor crítico para el éxito escolar en todos los niveles. En un artículo sobre las aspiraciones de carrera de EPG, Raque-Bogdan y Lucas (2016) mencionan que los EPG tienen muchas dificultades, especialmente relacionadas con asuntos familiares y económicos. Regularmente, los EPG viven con sus padres, los cuales no cuentan con experiencia académica, de ahí que no puedan ayudar eficazmente a sus hijos.

En tal sentido, en el estudio de Castillo y Cabezas (2010) se señala que hay más EPG matriculados en la educación superior cuando están presentes ambos padres en el hogar, tal vez por la motivación que pueden ejercer en sus hijos. De hecho, la influencia es mayor cuando uno o ambos padres cuentan con estudios superiores, pues hay mayor orientación y motivación hacia la cultura universitaria (Linne, 2018).

El hecho que los padres de los EPG no tengan un grado académico impacta en su capital social y cultural, ya que son quienes transfieren esos conocimientos y habilidades (Tello y Lonn, 2017). Regularmente, los EPG tienden a tener dificultades para relacionarse social y culturalmente en la universidad, y normalmente no conversan con sus padres sobre cuestiones académicas (Flanagan, 2017). Según Donoso, Donoso y Arias (2010), el compromiso de los padres con la educación de sus hijos resulta esencial para que estos, desde temprana edad, puedan imaginarse un proyecto escolar y profesional.

Sin embargo, en el caso de los EPG la realidad es distinta, pues carecen de orientación, motivación y apoyo para afrontar los retos de la universidad. En otras palabras, los EPG no tienen una referencia para apoyarse en su ingreso y trayecto en la educación superior (Soto Hernández, 2016). 

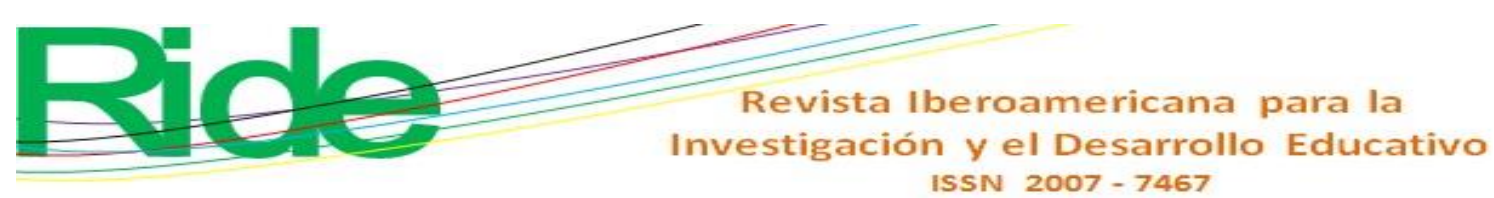

enfoque no atiende específicamente a los EPG. Por lo tanto, es imperativo que las universidades conozcan el perfil de los estudiantes, principalmente de los EPG.

Hicks y Wood (2016) mencionan que hace falta indagar en el conocimiento que tienen los docentes sobre los EPG para entenderlos y orientarlos mejor durante su proceso educativo, mientras que Flanagan (2017) considera que hace falta investigar sobre los EPG en Latinoamérica. Específicamente, se requieren investigaciones sobre EPG en el contexto mexicano, pues son inexistentes. La mayoría de los estudios sobre EPG son realizados en EE. UU. (Clemens, 2016; Hicks y Wood, 2016; Ishitani, 2016; Katrevich y Aruguete, 2017; Le et al., 2016; Means y Pyne, 2017; Mwaikinda y Aruguete, 2016). De hecho, solo en Argentina y Chile se ha indagado sobre este asunto (Castillo y Cabezas, 2010; Flanagan, 2017; Linne, 2018; Soto Hernández, 2016).

Por eso, con este estudio se pretende ofrecer un insumo literario sobre los EPG en el contexto de universidades mexicanas, en especial sobre sus historias de vida para entender su trayectoria escolar. Se espera que con los resultados de este trabajo las autoridades académicas, los psicólogos educativos y los docentes-tutores puedan comprender mejor las características, las experiencias y las motivaciones de los EPG. De hecho, al valorar las historias de vida de los estudiantes exitosos se pueden identificar características y habilidades que sirvan como base para el diseño de estrategias dirigidas a EPG en riesgo de deserción.

En concreto, las preguntas de investigación formuladas fueron las siguientes:

a. ¿Cuáles han sido las historias de vida de los estudiantes exitosos de primera generación en educación superior?

b. ¿Cómo se definen a sí mismos los estudiantes exitosos de primera generación?

c. ¿Cuáles han sido las barreras que han enfrentado los estudiantes exitosos de primera generación en la educación superior?

d. ¿Cuáles han sido los sistemas de apoyo para los EPG?

\section{Metodología}

Se utilizó un método cualitativo con enfoque narrativo. De acuerdo con Patton (2015), el enfoque narrativo se centra en las historias contadas por las personas para capturar la experiencia vivida de la gente en relación con su propio significado. Creswell (2014), por su parte, menciona que este enfoque analiza la vida de los individuos para después ser recontada por el investigador. En otras palabras, examina la vida humana a través de la narrativa (Arias-Cardona y AlvaradoSalgado, 2015), lo que permite el acceso al conocimiento (Clandinin y Connelly, 2000). Con los 

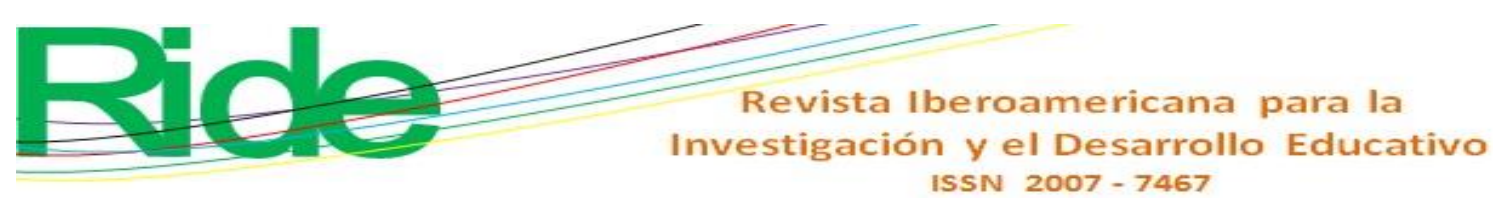

enfoques narrativos de investigación se procura proveer una noción de la realidad individual, y no colectiva, a través de las experiencias de los participantes que son representadas en sus narraciones (Bold, 2012).

\section{Participantes y muestra}

Los participantes para este estudio narrativo fueron EPG mexicanos de educación superior que lograron con éxito obtener un título universitario. La muestra que se utilizó fue no probabilística de tipo intencional (Check y Schutt, 2012). Específicamente, se utilizó una estrategia de muestreo caso típico, dado que los participantes son casos con criterios en común (Patton, 2015). Fueron cuatro participantes (una mujer y tres hombres) quienes lograron integrarse social y culturalmente en el ambiente universitario y que demostraron diversas habilidades y actitudes que les permitieron completar su trayectoria universitaria. En la tabla 1 se muestra una descripción más detallada de los participantes.

Tabla 1. Características demográficas de los participantes

\begin{tabular}{|l|l|l|l|l|}
\hline Seudónimo & Género & Edad & Programa educativo & Nivel educativo (padres) \\
\hline Oscar & Hombre & $21-31$ & Licenciatura en Psicología & Secundaria (ambos) \\
\hline Karen & Mujer & $32-42$ & Ingeniería en Telemática & Primaria (madre) \\
\hline Javier & Hombre & $22-32$ & $\begin{array}{l}\text { Licenciatura en Tecnología } \\
\text { Educativa }\end{array}$ & Bachillerato (ambos) \\
\hline Raúl & Hombre & $21-31$ & $\begin{array}{l}\text { Licenciatura en } \\
\text { Mercadotecnia Digital }\end{array}$ & Primaria (ambos) \\
\hline
\end{tabular}

Fuente: Elaboración propia

El participante Oscar estudio fuera de su ciudad de origen: vivió con sus amigos con los cuales rentaba una casa. Los padres de Oscar cubrían sus gastos dado que él no trabajaba (solo estudiaba). En cuanto a la participante Karen, también estudió en una ciudad de donde no era oriunda, por lo que vivía en casa de asistencia donde le proveían alimentos y una habitación. Ella tenía que trabajar como vendedora para pagar la renta, y se apoyaba económicamente de su madre. Javier, por su parte, vivía con su padre y madre en la ciudad donde estudió; aunque tenía beca por su promedio alto, trabajaba y se apoyaba económicamente de sus padres para solventar gastos. $\mathrm{Al}$ igual que el participante Javier, Raúl vivía con sus padres en la misma ciudad donde estudió y trabajaba en un banco para costear sus estudios. 

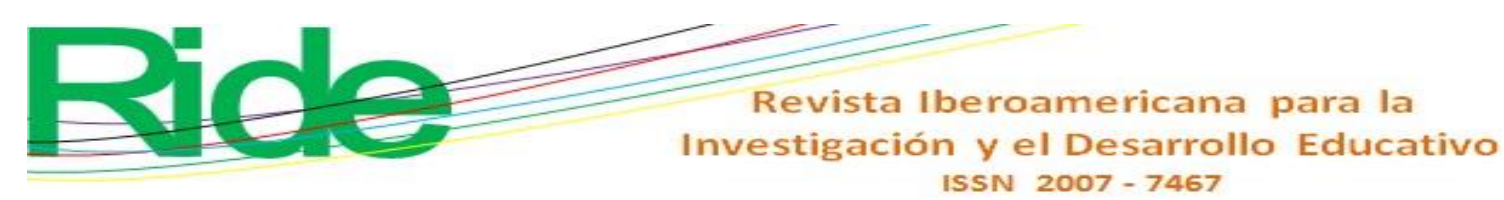

Empecé a ver lo que era el plan académico y vi... una escuela privada, y miraba que... [en mi universidad] ya estaban teniendo lo que es la internacionalización, cosa que la privada no encontré nada de eso, entonces fue lo que también me llamó más la atención.

Oscar no tenía la seguridad de cuál carrera estudiar, pero sabía tenía que estar relacionada con la medicina. Explicó que su motivación era "tener... título universitario y porque... decía voy a ser el primero en tener un título, siempre [pensaba] quiero terminar una carrera", aunque en ocasiones dudaba sobre en cuál área enfocarse. Oscar mencionó que "estando en la universidad,... [la] carrera era muy amplia... [y] tenía muchas áreas de estudio". Entonces "íbamos avanzando por carreras y había algo nuevo". Es decir, Oscar dudaba en qué especializarse porque "avanzaba un poquito más y miraba que había otros temas".

En la universidad su experiencia fue agradable; era un estudiante que no faltaba a la escuela y pasaba momentos alegres: "Con mis amigos se fomentó como una hermandad, se podría decir, porque estábamos siempre juntos". Su intención era divertirse y conocer personas. Aunque dijo que "no era de los considerados nerds, era tranquilo y aprovechaba el tiempo". Académicamente no sentía presión y no se estresaba, menos por cuestiones económicas. Oscar dijo que "cuando fue representante de grupo en la universidad no pagaba inscripción". Haber sido electo para ese cargo le ayudó económicamente. Oscar mencionó:

Yo [cuando] entré me eligieron como representante, me dijeron bueno tú tienes un derecho o un premio, no sé, se podría decir, un estímulo a seguir siendo representante de grupo. Gracias a Dios, mis padres me apoyaron económicamente, no me faltó nada.

Si bien como estudiante universitario no experimentaba estrés por cuestiones académicas o económicas, Oscar comentó que "hubo momentos donde no quería seguir estudiando porque me daba flojera". Sin embargo, lo que lo motivó a seguir - a pesar de los contratiempos - fueron sus amigos y docentes. Oscar recordó una experiencia con una profesora:

Una maestra era muy especial, muy difícil de tratar con ella; si no se hacía lo que ella decía al pie de la letra no te revisaba... Hasta que ella llegaba, se sentaba y sacaba su café, decía ahora sí, ya te puedo revisar, no te revisaba hasta que llegaba.

A pesar de la situación mencionada, Oscar la recuerda como una persona importante en su experiencia universitaria, dado que "le gustó aprender de ella". En relación con sus amigos, comentó que los "momentos de convivencia con los compañeros" fueron momentos alegres. En 


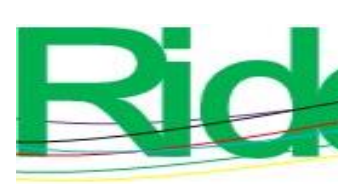

Revista Iberoamericana para la Investigación y el Desarrollo Educativo ISSN 2007 - 7467

su época de universitario se enfocó en fomentar lazos de amistad con sus compañeros con quienes vivía. Pero “de la familia me despegué porque venía [a casa de mis padres] una vez al mes”.

En la actualidad sigue considerando a sus compañeros como amigos, pues está pendiente de sus vidas personales y profesionales. A pesar de que la experiencia universitaria de Oscar fue positiva, también hubo momentos en los que experimentó tristeza, enojo e ira. Sin embargo, sus características y emociones le permitieron persistir y nunca rendirse. El impulso interno de querer un título universitario y su responsabilidad lo mantuvieron motivado para salir adelante. Oscar mencionó: "Yo digo que mi personalidad es cambiante". Esa personalidad y sus características como ser "extrovertido, creativo y serio cuando fuera necesario" contribuyeron a que fuera un estudiante exitoso.

La universidad en sí también influyó en la motivación de Oscar, pues sus profesores y la forma de enseñar a través de casos le ayudaron a despertar interés en él: "Los maestros planteaban casos fuertes que nos ayudaban a desarrollar el ojo clínico”. Como estudiante exitoso de primera generación ha sido de gran influencia en su familia: "Mi hermana y primos también se han graduado de la universidad desde que salí yo".

\section{La historia de Karen}

La experiencia universitaria de Karen fue "gratificante... una experiencia grandiosa". Ella la recuerda con mucha alegría porque tuvo la oportunidad de estudiar, practicar y aplicar lo que aprendía en los laboratorios de la universidad. Karen dijo que se "la pasaba en la universidad, en el laboratorio arreglando equipos, instalaba redes e iba a clases en la mañana y en la tarde". Haber estudiado en una escuela preparatoria de la universidad le ayudó a tomar la decisión de quedarse, pues algunos de sus compañeros también asistieron a la misma universidad y facultad. Karen mencionó que "eran buenos, amables y había disponibilidad para hacer equipo, compartían y eran cálidos". En cuanto a los docentes, ella recuerda su relación con ellos "muy formal, seria... [en] el rol de cada quien".

Karen normalmente comía en la escuela, en las cafeterías de la universidad, y en la noche cenaba en la casa donde vivía con sus compañeras. En algún tiempo compartió cuarto y momentos agradables en los que platicaban sobre sus situaciones diarias y se daban apoyo. Vivían en un ambiente de mucha confianza. Ella las recuerda como "experiencias diferentes", pues había “cercanía entre las compañeras y mucha confianza". 


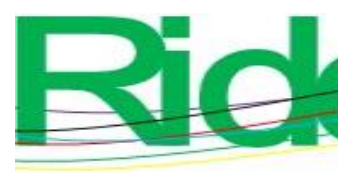

Revista Iberoamericana para la Investigación y el Desarrollo Educativo ISSN $2007-7467$

En cuanto a sus compañeros de clase, la mayoría eran hombres, quienes eran amables y tenían disponibilidad para apoyar. Karen comentó "que siempre había disponibilidad para hacer equipo, eran compartidos y cálidos”. Actualmente, Karen y sus excompañeros todavía interactúan a través de las redes sociales.

Karen siempre sintió el apoyo y motivación de su novio y su familia. Aunque mencionó que "había una economía limitada, pero no como para no terminar la carrera". Específicamente, tuvo el apoyo de su mamá y de sus tíos profesionistas. Karen compartió que "su familia es muy motivadora". En la experiencia vivida en la universidad, Karen también recibió el apoyo de sus maestros, a los cuales recuerda como "buenos y acogedores".

Aunque la universidad en la que estudió no era su primera opción, le aportó experiencias enriquecedoras que le ayudaron a cumplir con sus metas. Desde el inicio, Karen tenía claro qué quería profesionalmente: "Mi pensamiento en ese entonces era estudiar, seguirme preparando, pues siempre tuve esa imagen de poder alcanzar mis metas”. Su visión siempre estuvo presente y también fue "muy observadora" y "dedicada, motivada" y con "dominio de las cosas". La capacidad de crearse una visión y mantenerse motivada intrínsecamente hizo de Karen una estudiante exitosa. Así como fue influenciada por sus tíos profesionistas y el apoyo de su madre, ella también ejerció influencia en su hermana para que lograra obtener un título universitario.

\section{La historia de Javier}

Javier recientemente se graduó con el promedio más alto de su generación. Cuando ingresó a la universidad no estaba seguro de la carrera que elegiría, pues realmente quería era estudiar cine. El comentó:

Yo quería estudiar cine... era mi sueño, mi plan era terminar la carrera y estudiar algo que me gustara. No me llamaba la atención la carrera de tecnología educativa, se me hacía muy equis, quería estudiar otra cosa.

Estudiar tecnología educativa para él, en ese entonces, era una oportunidad de obtener un título para después estudiar lo que realmente quería. El mencionó: "Yo siempre vi a la educación o ir a estudiar como algo que tenía que hacer, por lo que hacer tareas no se me hizo complicado". A pesar de sus dudas al inicio como universitario, su experiencia fue de "estrés, alegría y satisfacción. He descubierto que siempre los primeros dos años o tres años soy muy tranquilo y en el último me suelto bastante", por lo que recuerda que su experiencia fue "enriquecedora". 

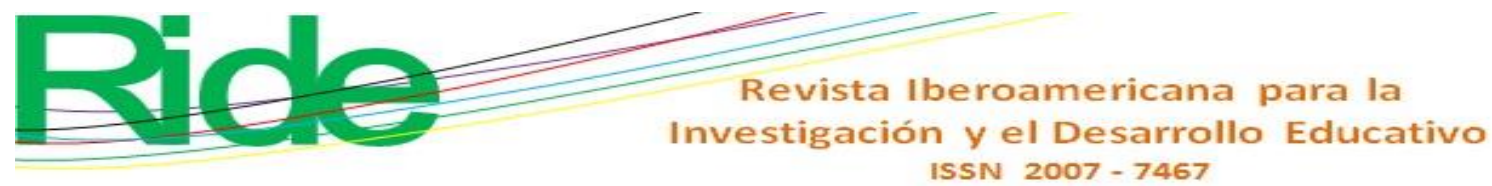

apoyo. Raúl recordó que se "apoyaban mucho, [se daban] todo tipo de apoyo y pues seguimos compartiendo en la actualidad".

Las limitaciones que tuvo Raúl en su trayectoria en la universidad tienen relación con cuestiones económicas e inseguridad social, pues "la mayoría de los apoyos fueron por parte de la escuela, ya que tenía una beca mensual”. Aunque "el apoyo de mi papá y mamá también me ayudó mucho", al igual que la información que la universidad ofrecía. Raúl fue una influencia positiva para sus hermanos, quienes también estudiaron una carrera universitaria.

\section{Discusión}

Las historias de Oscar, Karen, Javier y Raúl tienen en común aspectos que abarcan más que sus características de EPG exitosos. En la figura 1 se muestran estas similitudes en categorías conceptuales, que derivaron del análisis de los datos.

Figura 1. Características de los EPG exitosos

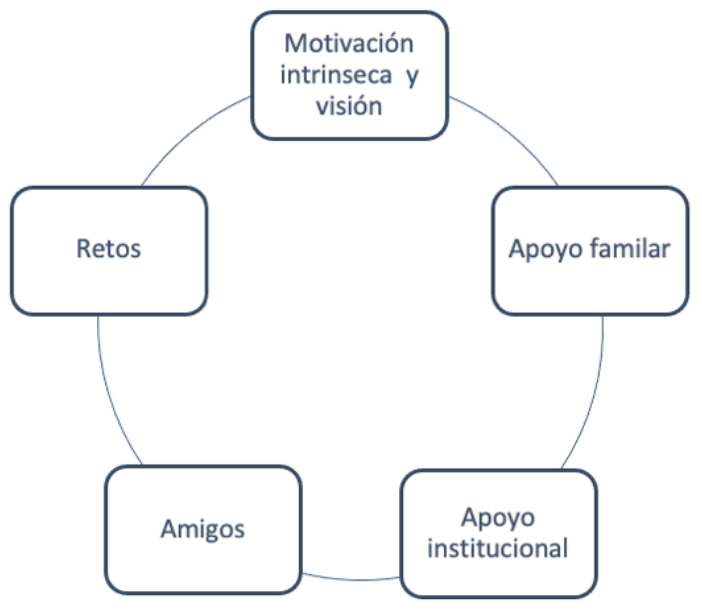

Fuente: Elaboración propia

Para Oscar, Karen, Javier y Raúl ser un estudiante universitario era un reto que implicaba superar muchas pruebas. Específicamente, las carencias económicas a las que todos se enfrentaron durante su trayectoria escolar. Al respecto, Linne (2018) menciona que para los estudiantes contar bajos recursos es determinante en su éxito o fracaso porque afecta las emociones y la motivación. Sin embargo, cabe resaltar que la motivación intrínseca y la visión mantuvieron a estos alumnos dispuestos a superar todos los retos. Querer lograr una carrera universitaria era la prioridad sin importar o preguntarse cómo lograrían lo que tenían en mente. 


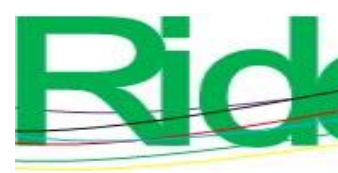

Revista Iberoamericana para la Investigación y el Desarrollo Educativo ISSN 2007 - 7467

Jacobson-Lundeber (2016), Tello y Lonn (2017), Shumaker y Wood (2016) y Soria et al. (2015) coinciden en que se requiere inteligencia emocional para tener la capacidad de visualizar el futuro. Oscar, Karen, Javier y Raúl supieron gestionar sus emociones, pues a pesar de enfrentar situaciones de estrés, supieron cómo manejar las situaciones difíciles, lo que demuestra la aseveración de Montiel (2017) sobre la capacidad de resiliencia de los EPG exitosos para superar los retos a pesar de las adversidades.

En relación con el apoyo familiar y de amigos, en las historias de los estudiantes se aprecia que ambos factores son claves para lograr el éxito. Si bien Raque-Bogdan y Lucas (2016) argumentan que las familias de EPG no tienen experiencia académica para apoyarlos, en muchos casos (como en los reseñados) resulta más significativo el apoyo moral. Por ejemplo, en la historia de Raúl, su mayor motivación fue mejorar la calidad de vida de sus padres.

Por otro lado, en cuanto a los amigos, Means y Pyne (2017) comentan que las interacciones entre compañeros crean un sentimiento de conexión y de pertenencia, lo que ayuda a los estudiantes a sentirse parte del grupo.

Vale acotar, sin embargo, que las interacciones de los estudiantes con los docentes no se dan de la misma manera (Mwaikinda y Aruguete, 2017), aunque en el caso de los EPG exitosos sí se percibió una relación estrecha con sus profesores. En este sentido, cabe resaltar que el apoyo de las instituciones educativas es importante para los EPG, pues las historias de Oscar, Javier, Karen y Raúl revelaron que la universidad fue un facilitador en la trayectoria académica por el apoyo brindado.

Los EPG exitosos que fueron entrevistados se definen como estudiantes con una motivación intrínseca y con una visión clara de lo que quieren para ellos y sus familias. Los EPG exitosos tienen bien definido sus fortalezas y debilidades emocionales, lo que les permite sortear los retos que se van presentando.

Las barreras de los EPG tienen relación con cuestiones económicas y de apoyo académico por parte de la familia. En algunas situaciones las emociones pudieran ser una barrera; sin embargo, la motivación demostrada en sus narraciones demostró ser determinante para lograr sus metas. Por lo tanto, los facilitadores de los EPG exitosos son la persistencia, la motivación, la capacidad de integrarse con sus amigos y su capacidad de visualizar una vida mejor. Los sistemas de apoyo morales de los EPG exitosos son sus familias y sus amigos, aunque la universidad también jugó una parte importante en el proceso (específicamente en la historia de Javier, pues fue quien recibió becas). No obstante, en las historias de Oscar, Karen y Raúl no se percibió un apoyo claro y conciso del sistema universitario, sino solo de los docentes y compañeros. 


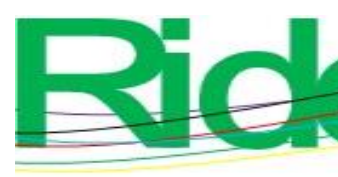

Revista Iberoamericana para la Investigación y el Desarrollo Educativo ISSN $2007-7467$

Las limitaciones del estudio fueron sucesos posiblemente deformados y olvidos provocados tal vez por la edad o algún trauma; sin embargo, a través de las preguntas durante la entrevista se intentó provocar los recuerdos vividos.

\section{Conclusiones}

La educación superior a nivel mundial enfrenta múltiples retos en distintas dimensiones. Uno de ellos es ofrecer a la población estudiantil una educación adecuada a sus necesidades y características. Los EPG tienen características diferentes que dificulta a las universidades brindar una educación de calidad y pertinente a la demanda estudiantil. Existe una amplia investigación sobre los EPG y los factores que influyen en el fracaso escolar; sin embargo, desde la perspectiva de estudiantes exitosos, las indagaciones son limitadas en México y en América Latina.

El propósito de este estudio cualitativo, por tanto, fue conocer las experiencias de estudiantes exitosos de primera generación en educación superior a través de la narración de sus historias de vida. Si bien no es un estudio exhaustivo, se explora el tema en el contexto mexicano con el fin de comprenderlo y compararlo con otros contextos para hallar posibles soluciones. La trayectoria académica de los EPG en la universidad supone barreras y facilitadores. Las barreras para los estudiantes tienen relación con su inexperiencia en la cultura universitaria y la falta de apoyo académico por parte de su familia, así como la incertidumbre y las emociones como el estrés, la ira o la desesperación. A pesar de ello, las historias narradas de los participantes demuestran que su motivación y resiliencia les facilitó lograr sus metas académicas.

La capacidad de los EPG de visualizar su futuro los incentivó a integrarse con compañeros y docentes para estudiar y trabajar en equipo. Sin embargo, aunque institucionalmente hay cierto apoyo, todavía se requieren programas universitarios específicos que faciliten la vida académica de los EPG, pues a pesar de su capacidad de sobreponerse a los retos, existe un número elevado que no culmina su objetivo, por lo que cabe preguntarse cuál es el rol de las instituciones de educación superior ante ese escenario. 


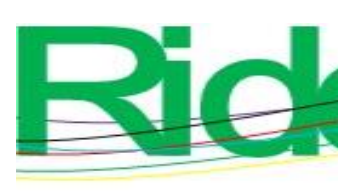
Revista Iberoamericana para la
Investigación y el Desarrollo Educativo
ISSN $2007-7467$

Futuras líneas de investigación

En futuros estudios se podrían explorar los programas universitarios que impactan directamente en el desarrollo o fomento de la capacidad de resiliencia, motivación y visualización, así como en las experiencias de estudiantes en riesgo de deserción. También sería conveniente estudiar el desempeño e impacto que tienen los programas universitarios existentes encargados de generar una cultura universitaria integradora.

\section{Referencias}

Arias-Cardona, A. M. y Alvarado-Salgado, S. V. (2015). Investigación narrativa: apuesta metodológica para la construcción social de conocimientos científicos. Revista CES Psicología, 8(2), 171-181.

Barraza-López, R. and Gonzalez (2016). Academic achievement and self-perception of multiple intelligences and emotional intelligence in first-generation college. Actualidades Investigativas en Educación, 16(2), 269-292.

Bold, C. (2012). Using narrative in research. London: Sage Publications.

Castillo, J. y Cabezas (2010). Caracterización de jóvenes primera generación en educación superior. Nuevas trayectorias hacia la equidad educativa. Calidad en la Educación, (32), 44-76.

Check, J. and Schutt, R. K. (2012). Research methods in education. London: Sage Publications.

Clandinin, D. J. and Connelly, F. M. (2000). Narrative inquiry: Experience and story in qualitative research. United States: Jossey-Bass

Clemens, R. F. (2016). Transitioning from high school to college: Examining the sources and influences of social capital for a first-generation Latina student. The Qualitative Report, 21(11), 2044-2072.

Creswell, J. W. (2014). Research design: Qualitative, quantitative, and mixed methods approaches. London: SAGE Publications.

Dennis, M. J. (2012). Anticipatory enrollment management: Another level of enrollment management. College and University, 88(1), 10-16.

Donoso, S., Donoso, G. y Arias, Ó. (2010). Iniciativas de retención de estudiantes de educación superior. Calidad en la Educación, (33), 15-61.

Flanagan, A. (2017). Experiencias de estudiantes de primera generación en universidades chilenas: Realidades y desafíos. Revista de la Educación Superior, 46(183), 87-104. Recuperado de http://resu.anuies.mx/ojs/index.php/resu/article/view/27 

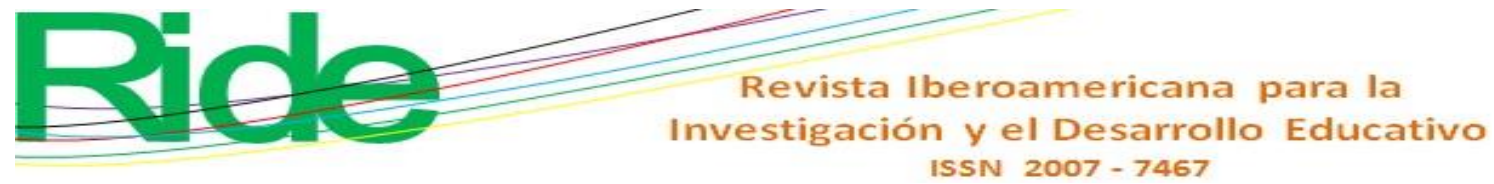

Patton, M. Q. (2015). Qualitative Research \& Evaluation Methods: Integrating Theory and Practice ( $4^{\text {th }}$ ed.). London: Sage Publications.

Raque-Bogdan, T. and Lucas, M. S. (2016). Career aspirations and the first-generation student: Unraveling the layers with social cognitive career theory. Journal of College Student Development, 57(3), 248-262. Doi: https//doi.org/10.1353/csd.2016.0026

Saldaña, J. (2016). The coding manual for qualitative researchers. London: Sage Publications.

Shumaker, R. and Wood, J. L. (2016). Understanding first-generation community college students: An analysis of covariance examining use of access to, and efficacy regarding institutionally offered services. The Community College Enterprise, 22(2), 9-17.

Soria, K. M., Roberts, J. E. and Reinhard, A. P. (2015). First-year college students' strengths awareness and perceived leadership development. Journal of Student Affairs Research and Practice, 52(1), 89-103. Doi: https//doi.org/10.1080/19496591.2015.996057

Soto Hernández, V. J. (2016). Estudiantes de primera generación en Chile: una aproximación cualitativa a la experiencia universitaria. Revista Complutense de Educación, 27(3), 11571173. Doi: https://doi.org10.5209/rev_RCED.2016.v27.n3.47562

Tello, A. M. and Lonn, M. R. (2017). The role of high school and college counselors in supporting the psychosocial and emotional needs of latinx first-generation college students. The Professional Counselor, 7(4), 349-359. Doi: https//doi.org/10.15241/amt.7.4.349 


\begin{tabular}{|c|c|}
\hline Rol de Contribución & Autor (es) \\
\hline Conceptualización & Enrique Bonilla Murillo \\
\hline Metodología & Enrique Bonilla Murillo \\
\hline Software & $\begin{array}{l}\text { Enrique Bonilla Murillo, Verónica Sagnité Solís Herebia. } \\
\text { «igual» }\end{array}$ \\
\hline Validación & $\begin{array}{l}\text { Enrique Bonilla Murillo, Verónica Sagnité Solís Herebia } \\
\text { «igual» }\end{array}$ \\
\hline Análisis Formal & $\begin{array}{l}\text { Enrique Bonilla Murillo, Verónica Sagnité Solís Herebia } \\
\text { «igual» }\end{array}$ \\
\hline Investigación & $\begin{array}{l}\text { Enrique Bonilla Murillo, Verónica Sagnité Solís Herebia } \\
\text { «igual» }\end{array}$ \\
\hline Recursos & $\begin{array}{l}\text { Enrique Bonilla Murillo, Verónica Sagnité Solís Herebia } \\
\text { «igual» }\end{array}$ \\
\hline Curación de datos & $\begin{array}{l}\text { Enrique Bonilla Murillo, Verónica Sagnité Solís Herebia } \\
\text { «igual» }\end{array}$ \\
\hline $\begin{array}{l}\text { Escritura - Preparación del } \\
\text { borrador original }\end{array}$ & $\begin{array}{l}\text { Enrique Bonilla Murillo «principal», Verónica Sagnité Solís } \\
\text { Herebia }\end{array}$ \\
\hline $\begin{array}{l}\text { Escritura - Revisión y } \\
\text { edición }\end{array}$ & $\begin{array}{l}\text { Enrique Bonilla Murillo, Verónica Sagnité Solís Herebia } \\
\text { «igual» }\end{array}$ \\
\hline Visualización & $\begin{array}{l}\text { Enrique Bonilla Murillo, Verónica Sagnité Solís Herebia } \\
\text { «igual» }\end{array}$ \\
\hline Supervisión & $\begin{array}{l}\text { Enrique Bonilla Murillo, Verónica Sagnité Solís Herebia } \\
\text { «igual» }\end{array}$ \\
\hline Administración de Proyectos & $\begin{array}{l}\text { Enrique Bonilla Murillo, Verónica Sagnité Solís Herebia } \\
\text { «igual» }\end{array}$ \\
\hline Adquisición de fondos & $\begin{array}{l}\text { Enrique Bonilla Murillo, Verónica Sagnité Solís Herebia } \\
\text { «igual» }\end{array}$ \\
\hline
\end{tabular}

